\title{
Evaluation of Hyponatremia among Psychiatric Inpatients: A Preliminary Local
} Appraisal

Saeed Shoja Shafti ${ }^{*}$, Alireza Memarie, Masomeh Rezaie, Masomeh Hamidi

University of Social welfare and Rehabilitation sciences, Razi Psychiatric Hospital, Tehran, Iran

* Corresponding Author: Saeed Shoja Shafti, Professor of Psychiatry, University of Social welfare and Rehabilitation sciences, Razi Psychiatric Hospital, Tehran, Iran. E-mail: ssshafti@gmail.com

Received date: November 18, 2019; Accepted date: December 26, 2019; Published date: December 30, 2019

Citation: Shoja Shafti S., Memarie A., Rezaie M., Hamidi M. (2019) Evaluation of Hyponatremia Among Psychiatric Inpatients: A Preliminary Local Appraisal, J Neurodegeneration and Neurorehabilitation. 2(1); Doi: 10.31579/2692-9422/004

Copyright: @ 2019 Saeed Shoja Shafti. This is an open-access article distributed under the terms of The Creative Commons Attribution License, which permits unrestricted use, distribution, and reproduction in any medium, provided the original author and source are credited.

Abstract:
Introduction: Hyponatremia is one of the most frequent ion and water disorders and severe hyponatremia is associated with
well-known clinical symptoms and manifestations. In the present assessment the incidence and clinical profile of hyponatremia
have been probed among a great sample of non-western psychiatric inpatients and compared with the available data in literature
regarding prevalence and other associated clinical characteristics.
Methods: All inpatients with idiopathic hyponatremia during the last sixty-four months had been included in the present study.
Clinical diagnosis, as well, was in essence based on 'Diagnostic and Statistical Manual of Mental Disorders, 5th edition (DSM-
5)'. Statistical significance had been defined as a p value $\leq 0.05$.
Results: While the annual incidence of hyponatremia in current evaluation was around $0.01 \%$, the annual incidence of mortality due
to hyponatremia was around $0.001 \%$. It was significantly more prevalent among male psychiatric inpatients (p<0.04) and patients
with duration of illness in excess of one year ( $<0.04)$. Furthermore it was meaningfully more evident among schizophrenic
patients (p<0.007), in comparison with remaining primary psychiatric disorders. There was no significant relationship between
hyponatremia and symptomatic profile, or serum level of sodium and occurrence of seizure. .
Conclusion: Hyponatremia was significantly more prevalent among male patients and cases with duration of illness in excess
of one year. Furthermore it was meaningfully more evident among schizophrenic patients.
Key words: hyponatremia; psychiatric disorders; psychotropic drugs; schizophrenia.

\section{Introduction}

Hyponatremia (serum sodium concentration $<136 \mathrm{mEq} / \mathrm{L}$ ) is one of the most frequent ion and water disorders. It is generally due to disproportionate renal water retention. Severe hyponatremia $(<125 \mathrm{mEq} / \mathrm{L})$ is associated with wellknown clinical symptoms and manifestations. However, even mild reductions in sodium blood levels have been shown to be associated with increased mortality and with the risk of falls and fractures. The diagnosis of hyponatremia, although requiring simple clinical and laboratory tests, may be complex and difficult [1]. Hyponatremia is a prevalent and potentially dangerous medical comorbidity in psychiatric patients, too [2]. Hyponatraemia can occur in the context of water intoxication, where water consumption exceeds the maximal renal clearance capacity along with a low serum and urine osmolality. Cross-sectional studies of chronically ill, hospitalised psychiatric patients have found the prevalence of water intoxication to be approximately 5\% [3]. It may occur, as well, due to druginduced syndrome of inappropriate antidiuretic hormone (SIADH), where the kidney retains an excessive quantity of solute-free water. In this situation, serum osmolality is low and urine osmolality is relatively high. The prevalence of SIADH has been estimated to be as high as $11 \%$ in acutely ill psychiatric patients [4]. Risk factors for antidepressant induced SIADH (increasing age, female gender, medical co-morbidity and polypharmacy) seem to be less relevant in the population of patients treated with antipsychotic drugs [5]. Overall prevalence of antipsychotic-induced hyponatraemia has been estimated at $0.004 \%$ [6] and $26.1 \%$ [5] of patients. Mild to moderate hyponatraemia presents as confusion, nausea, headache and lethargy. As the plasma sodium falls, these symptoms become increasingly severe and seizures and coma can develop. So while monitoring of plasma sodium is desirable for all those receiving antipsychotics, signs of confusion or lethargy should provoke thorough diagnostic analysis, including plasma sodium determination and urine osmolality (3). Prevalence of polydipsia in a population of chronic psychiatric patients can be as high as 6 to $17 \%$ [7]. Schizophrenia represents $80 \%$ of cases reported [8]. Early onsets of psychiatric disorder and long duration of that or poor response to psychopharmacotherapy have been identified as significant risk factors for appearance of hyponatremia [9]. Patients with hyponatremia may be asymptomatic or present with nausea, anorexia, muscle cramps, weakness, fatigue, confusion and disorientation. Severe hyponatremia may result in serious neurologic sequelae such as coma and seizures, and death. Advanced age, too, appears to be a risk factor for this adverse effect, as does the concomitant use of diuretics [10]. Like other psychotropic medications, it is suspected that atypical antipsychotics can induce hyponatremia by either stimulating antidiuretic hormone release from the brain or enhancing antidiuretic hormone activity in the kidneys [10]. Currently, there are no reliable estimates of incidence or risk of hyponatremia from atypical antipsychotic drugs in older adults [11]. On the other hand, while acute hyponatremia is characterized by onset of symptoms in less than $48 \mathrm{~h}$, chronic hyponatremia develops over $48 \mathrm{~h}$ and most patients have chronic hyponatremia. The serum sodium concentration is usually above $120 \mathrm{meq} / \mathrm{L}$. Brain adapts itself to hyponatremia by generation of idiogenic osmoles. This is a protective mechanism that reduces the degree of cerebral edema; it begins on the first day and is complete within several days. Hence in chronic hyponatremia patients may appear asymptomatic [12]. Mild chronic hyponatremia is not benign as previously thought and can directly contribute to increased morbidity and possibly, mortality $[13,14]$. In the present assessment the incidence and clinical profile of hyponatremia have been 
probed among a great sample of non-western psychiatric inpatients and compared with the available data in literature regarding prevalence and other associated clinical characteristics.

\section{Methods}

Razi psychiatric hospital in south of capital city of Tehran, as one of the largest and oldest public psychiatric hospitals in the Middle East, which has been established formally in 1917 and with a capacity around 1375 active beds, had been selected as the field of study in the present retrospective assessment. For evaluation, all inpatients with idiopathic hyponatremia during the last sixty-four months had been included in the present study. Hyponatremia had been detected during systematic baseline laboratory checkups or later as a result of clinician's request and examination due to various symptoms or signs. Clinical diagnosis, as well, was in essence based

On 'Diagnostic and Statistical Manual of Mental Disorders, 5th edition (DSM-5)' [15]. Among eighteen detected cases, twelve patients were male and six patients were female. Also, the necessary general permission had been obtained from the patients or their relatives during admission. The mean age of samples, as well, was around $43.92 \pm 9.51$ years old and 52.50 \pm 8.34 years old for male and female patients, respectively. Most of the patients met with diagnosis of schizophrenia $(n=13)$, and the remaining met with diagnosis of mental retardation $(n=1)$, schizoaffevtive $(n=1)$, bipolar disorder $(\mathrm{n}=2)$ and major depressive disorder $(\mathrm{n}=1)$ (Table 1$)$.

\begin{tabular}{|c|c|c|c|c|c|c|}
\hline Number & Gender & Age & Chronic & Diagnosis & Symptoms & Sodium ( mEq/L) \\
\hline $\mathbf{1}$ & M & 46 & + & Schizophrenia & Seizure & 119 \\
\hline $\mathbf{2}$ & M & 37 & - & Bipolar & Weakness & 121 \\
\hline $\mathbf{3}$ & F & 47 & + & Schizophrenia & Polydipsia & 122 \\
\hline $\mathbf{4}$ & M & 56 & + & Schizophrenia & Seizure & 114 \\
\hline $\mathbf{5}$ & F & 56 & + & Schizophrenia & Seizure & 119 \\
\hline $\mathbf{6}$ & M & 52 & + & Schizophrenia & Seizure, Polydispia & 122 \\
\hline $\mathbf{7}$ & F & 63 & + & Schizophrenia & Polydispia & 120 \\
\hline $\mathbf{8}$ & F & 37 & - & Bipolar & - & 126 \\
\hline $\mathbf{9}$ & M & 25 & - & Mental retardation & Headache, Nausea & 119 \\
\hline $\mathbf{1 0}$ & M & 46 & - & Schizophrenia & - & 132 \\
\hline $\mathbf{1 1}$ & M & 50 & + & Schizophrenia & - & 119 \\
\hline $\mathbf{1 2}$ & M & 57 & + & Schizophrenia & Dizziness & 124 \\
\hline $\mathbf{1 3}$ & M & 32 & - & Schizoaffective & - & 113 \\
\hline $\mathbf{1 4}$ & M & 34 & + & Schizophrenia & - & 126 \\
\hline $\mathbf{1 5}$ & M & 45 & - & Depression & - & 130 \\
\hline $\mathbf{1 6}$ & F & 56 & + & Schizophrenia & - & 116 \\
\hline $\mathbf{1 7}$ & M & 47 & - & Schizophrenia & Weakness & 127 \\
\hline $\mathbf{1 8}$ & F & 56 & + & Schizophrenia & polydipsia & 122 \\
\hline
\end{tabular}

Abbreviations: $\mathrm{M}=$ male; $\mathrm{F}=$ female; Chronic $=$ duration of illness more than one year. Table 1: Demographic and clinical characteristics or hyponatremic patients.

\section{Statistical analysis:}

Analysis of dependent variables had been accomplished by't-test' and appraisal of independent variables had been explored by means of 'comparison of proportions'. Statistical significance, as well, had been defined as a $p$ value $\leq 0.05$. Med Calc Statistical Software version 15.2 was used as statistical software tool for analysis.

\section{Results:}

As said by results, among 20118 psychiatric patients hospitalized in razi psychiatric hospital, during the last sixty-four months (April of 2014-August 2019), eighteen patients had been diagnosed as hyponatremic, whether symptomatic or asymptomatic, during their inpatient treatment, based on laboratory checkups. So, the annual incidence of hyponatremia in the current inpatients' evaluation was around $0.01 \%$. In spite of referral to intensive care unit and given medical treatment, one elderly chronic female patient died by reason of hyponatremia. Accordingly, in the present survey the annual incidence of mortality because of hyponatremia was around $0.001 \%$. Also, with regard to gender difference and in keeping with results, while there was no significant difference regarding age between male and female patients $(\mathrm{t}$ $=1.962, \mathrm{p}<0.08$, CI 95\%: $-18.29,1.13)$, hyponatremia was significantly more prevalent among male psychiatric inpatients $(\mathrm{z}=2.00, \mathrm{p}<0.04$, CI 95\%:0.006, 0.660). Besides, concerning chronicity of psychiatric disorders, comparison of proportions showed that hyponatremia was significantly more prevalent among psychiatric patients with duration of illness in excess of one year $(\mathrm{p}<0.04)$ (twelve patients in the chronic wards versus six patients in the acute districts of the hospital). Moreover, with respect to prevalence of primary psychiatric disorders among patients, who have experienced hyponatremia during the last 64 months, schizophrenia was significantly more prevalent in comparison with remaining disorders $(\mathrm{z}=2.66, \mathrm{p}<0.007, \mathrm{CI}$ 95\%: -0.11, 0.77). While, eleven patients had different clinical symptoms due to hyponatremia, seven cases were asymptomatic and diagnosis had been confirmed based on merely coincidental checkup. Quantitatively, analysis revealed no significant difference between those groups $(\mathrm{z}=1.33, \mathrm{p}<0.18$, CI 95\%: $-0.54,0.10)$. Also, though mean total plasma level of sodium was around $121.72 \pm 4.97 \mathrm{mEq} / \mathrm{L}$, there was no significant difference between serum level of sodium in seizure cases (mean total $=119.75 \pm 1.29 \mathrm{mEq} / \mathrm{L}$ ) and non-seizure patients (mean total $=122.64 \pm 5.06 \mathrm{mEq} / \mathrm{L})(\mathrm{t}=1.929, \mathrm{p}<0.28$, CI 95\%: $-6.07,0.29)$.

\section{Discussion:}

Many psychiatric patients have polydipsia and polyuria without identifiable underlying medical causes. Hyponatremia develops in some polydipsic patients and can progress to water intoxication with such symptoms as confusion, lethargy, psychosis, and seizures or death [16]. This syndrome is sometimes called "compulsive water drinking," "psychogenic polydipsia," and "self-induced water intoxication." Although the underlying pathophysiology of the syndrome is unclear, several factors have been implicated in producing polydipsia and symptomatic hyponatremia. These include a possible hypothalamic defect, the syndrome of inappropriate secretion of $\mathrm{ADH}$ (SIADH), and neuroleptic medication. Evaluation of psychiatric patients with polydipsia includes a search for other medical causes of polydipsia, polyuria, hyponatremia, and SIADH. Treatment modalities currently available include fluid restriction and medications [16]. Polydipsia, chronic or intermittent, with or without hyponatremia, frequently occurs among chronic patients with schizophrenia. The pathogenesis of polydipsia remains poorly understood. It has been suggested that maybe in 
some of these patients, polydipsia and hyponatremia are consequences of patients' adjustment to a prolonged intake of an insufficient diet, dominantly poor in potassium. Deficits of potassium, without significant hypokalemia, may cause impairment of the urine-concentrating ability with polyuriapolydipsia. A fall of intracellular tonicity, dominantly due to a decreased amount of $\mathrm{K}(+)$ and attendant anions in cells, should be accompanied with a fall of extracellular osmolality. Because of the diminished content of ions that may diffuse out of cells and because osmotic equilibrium between the extracellular fluid and intracellular fluid compartments cannot beestablished in a short period of time, these patients have a diminished abilityto adapt to an excessive intake of fluids. These mechanisms might be related to the development of polydipsia and water intoxication in patients with different mental and somatic disorders [17]. On the other hand, while mild chronic hyponatremia, as defined by a persistent ( $>72$ hours) plasma sodium concentration between 125 and $135 \mathrm{mEq} / \mathrm{L}$ without apparent symptoms, is common in ambulatory patients and generally perceived as being inconsequential [18], hyponatremia at time of inpatient admission is associated with increased severity of illness and mortality in patients hospitalized for treatment of medical conditions and should trigger enhanced clinical monitoring to identify and treat somatic disorders [19]. DrugInduced hyponatremia is a frequent and potentially seri $\neg$ ous adverse reaction with many psychopharmacological agents, mediated in most cases by SIADH. This condition most often leads to subtle psychomotor symptoms due to its slow progression, permitting a compensatory adjustment of intracellular volume in the central nervous system. Subtle psycho $\neg$ motor symptoms and motor imbalance readily resolve after discontinuation of the responsible pharmacological agent [19]. In contrast, rapid onset of hyponatremia may present with life-threatening encephalopathy, which requires emergent in $\neg$ fusion of intravenous hypertonic saline to reverse acute cerebral edema (20). Back to our discussion and along with analysis, while our estimate respecting prevalence of hyponatremia was lower than the approximations of Ohsawa et al. [9] Manu et al. [19], Sahay et al. [12], Meulendijks et al. [4] and Yang et al. [5], it was higher in comparison with the calculations of Letmaier et al. [6]. These variances can be due to diverse variables and confounding factors, like the principal of analysis, sample selection and settings of study. On the other hand, in spite of its acknowledgement as a known risk factor, a deficiency of epidemiological studies regarding hyponatremia is palpable, which is not limited to prevalence and incidence, too. Regarding the risk of mortality as a result of hyponatremia, our outcome, though limited to only one elderly and chronic female schizophrenic patient, was, more or less, in harmony with the conclusions of Naticchia et al. [1], Rondon-Berrios et al. [18]] and Siegel [2], who had indicated that, even mild reductions in sodium blood levels havebeen shown to be associated with increased mortality and hyponatremia should be regarded as a prevalent and potentially dangerous medical comorbidity in psychiatric patients. Moreover, consistent with the outcome of the present evaluation, male gender could be regarded as a risk factor for

\section{References:}

1. Naticchia A, Ferraro PM, D'Alonzo S, Gambaro G. [Hyponatremia: practical approach to diagnosis and treatment]. G Ital Nefrol 2011; 28(3):305-313.

2. Siegel AJ. Hyponatremia in psychiatric patients: update on evaluation and management. Harv Rev Psychiatry 2008; 16 (1):13-24.

3. Madhusoodanan S. Hyponatraemia associated with psychotropic medications. A review of the literature and spontaneous reports. Adverse Drug React Toxicol Rev 2002; 21:17-29.

4. Meulendijks D. Antipsychotic-induced hyponatraemia: a systematic review of the published evidence. Drug Saf 2010; 33:101-114.

5. Yang HJ. Antipsychotic use is a risk factor for hyponatremia inpatients with schizophrenia: a 15-year follow-up study. Psychopharmacology (Berl) 2017; 234:869-876.

6. Letmaier $\mathrm{M}$ et al. Hyponatraemia during psychopharmacological treatment: results of a drug surveillance programme. Int $\mathbf{J}$ occurrence of hyponatremis, at least among psychiatric patients. Concerning chronicity of psychiatric disorders, conclusion of the current appraisal was in accord with the suppositions of Ohsawa et al. [9] and Gandhi et al. [11], who had found a long duration of psychiatric disorder and a prolonged admission as statistically significant factors in occurrence of hyponatremia, though the later had specified that due to atypical antipsychotic medications among older patients. Moreover, with regard to higher prevalence of hyponatremia among schizophrenic patients, our conclusion was again in harmony with the findings of Ohsawa et al. [9], Lapierre et al. [7] and Siegel [2], who had found the aforesaid medical complication more among that disorders. Furthermore, regarding clinical profile of hyponatremia, our outcome, which had revealed that there was no significant difference, quantitatively, between symptomatic and asymptomatic hyponatremic cases, was in agreement with the report of Liu et al. [10], who stated that patients with hyponatremia may be asymptomatic or present with nausea, anorexia, muscle cramps, weakness, fatigue, confusion and disorientation. Besides, our result as regards the insignificant relationship between sodium levels and severity of clinical symptoms, like seizure, was somewhat in accord with the findings of Manu et al. [19], who had found medical deteriorations incidences in both hyponatremic and non-hyponatremic patients, although more among the first group. Anyhow, as the most common electrolyte abnormality in medical prac $\neg$ tice, recent evidence from meta-analyses indicates that hypo $\neg$ natremia is associated with increased morbidity and excess mortality, and psychiatric patients are at substan $\neg$ tial risk for this adverse event, which may occur with many pharmacologic agents. Since SIADH is the most common underly-ing mechanism, accounting for over $80 \%$ of cases in psychi $\neg$ atric patients, in contrast to less than $30 \%$ in general medi $\neg$ cal practice, clinicians prescribing pharmacological agents conferring risk for dilutional hyponatremia should main $\neg$ tain a high index of suspicion for the full spectrum of conse $\neg$ quent clinical severity [20]. Restricted period of study, due to inadequate registration and documents in the last decades, and thus insufficient number of cases with idiopathic hyponatremia, and no concurrent checking of osmolality of serum and urine for proper classification of hyponatremia into water intoxication and drug-induced syndrome of inappropriate antidiuretic hormone, do not permitgeneralization of outcomes to more than a preliminary survey. No doubt, further methodical studies in future will improve our clinical idea concerning diagnosis and management of this important medical problem among psychiatric patients.

\section{Conclusion}

Hyponatremia was significantly more prevalent among male patients and cases with duration of illness in excess of one year. Furthermore it was meaningfully more evident among schizophrenic patients.

Neuropsychopharmacol 2012; 15:739-748.

7. Lapierre E, Berthot BD, Gurvitch M, Rees I, Kirch DG.Polydipsia and hyponatremia in psychiatric patients: challenge to creative nursing care. Arch Psychiatr Nurs 1990; 4(2):87-92.

8. Assouly-Besse F, Seletti B, Lamarque I, Elghozi D, Petitjean F. ["Polydipsia, intermittent hyponatremia and psychoses" syndrome: a diagnosis and therapeutic management of a case]. Ann Med Psychol (Paris) 1996; 154(4):259-263.

9. Ohsawa H, Kishimoto T, Hirai M, Shimayoshi N, Matsumura K. et al. An epidemiological study on hyponatremia in psychiatric patients in mental hospitals in Nara Prefecture. Jpn J Psychiatry Neurol.1992;46(4):883-839.

10. Liu BA, Mittmann N, Knowles SR, Shearnh. hyponatremia and the syndrome of inappropriate secretion of antidiuretic hormone associated with the use of selective serotonin reuptake inhibitors: a review of spontaneous reports. can med assoc j 1996; 155 (5):519-527. 
11. Gandhi S, McArthur E, Reiss JP, Mamdani MM, Hackam DG. Atypical antipsychotic medications and hyponatremia in older adults: a population based cohort study. Canadian Journal of Kidney Health and Disease 2016; 3(21):1-11.

12. Sahay M, Sahay R. Hyponatremia: A practical approach. Indian Journal of Endocrinology and Metababolism 2014; 18(6): 760-771.

13. Rondon-Berrios H, Berl T. Mild Chronic Hyponatremia in the Ambulatory Setting: Significance and Management. Clin J Am Soc Nephrol 10(12): 2268-2278.

14. Hoorn EJ, Zietse R: Hyponatremia and mortality: Moving beyond associations. Am J Kidney Dis 2013; 62(149); 232- 244.

15. American Psychiatric Association (APA). Diagnostic and Statistical Manual of Mental Disorders. 5th ed. Washington, DC: American Psychiatric Association, 2013.

16. Illowsky BP, Kirch DG. Polydipsia and hyponatremia in psychiatric patients. Am J Psychiatry 1988; 145(6):675-683.
Margetić B, Aukst-Margetić B. A different hypothesis on hyponatremia in psychiatric patients: treatment implications and experiences. World J Biol Psychiatry 2009; 10(4):677-681.

18. Rondon-Berrios H, Berl T. Mild Chronic Hyponatremia in the Ambulatory Setting: Significance and Management. Clin J Am Soc Nephrol 2015;10(12):2268-2278.

19. Manu P, Ray K, Rein JL, De Hert M, Kane JM, Correll CU. Medical outcome of psychiatric inpatients with admission hyponatremia. Psychiatry Res 2012; 198(1):24-27.

20. Siegel A J, Forte SS, Bhatti NA, Gelda SE. Drug-Related Hyponatremic Encephalopathy: Rapid Clinical Response Averts LifeThreatening Acute Cerebral Edema. American Journal of Case Reports 2016; 17: 150-153.

17.

This work is licensed under Creative Commons Attribution 4.0 License
Ready to submit your research? Choose Auctores and benefit from:

* fast, convenient online submission

* rigorous peer review by experienced research in your field

* rapid publication on acceptance

* authors retain copyrights

* unique DOI for all articles

* immediate, unrestricted online access

At Auctores, research is always in progress.

Learn more https://www.auctoresonline.org/journals/neurodegenerationand-neurorehabilitation 